\title{
Radioimmunotherapy of fungal diseases: the therapeutic potential of cytocidal radiation delivered by antibody targeting fungal cell surface antigens
}

\author{
Joshua D. Nosanchuk ${ }^{1,2}$ * and Ekaterina Dadachova ${ }^{2,3}$ \\ 1 Department of Medicine, Albert Einstein College of Medicine, Bronx, NY, USA \\ 2 Department of Microbiology and Immunology, Albert Einstein College of Medicine, Bronx, NY, USA \\ ${ }^{3}$ Department of Nuclear Medicine, Albert Einstein College of Medicine, Bronx, NY, USA
}

\section{Edited by:}

Carlos Pelleschi Taborda, University of São Paulo, Brazil

Reviewed by:

Marcio Rodrigues, Federal University of Rio de Janeiro, Brazil

David Van Duin, Cleveland Clinic, USA

\section{*Correspondence:}

Joshua D. Nosanchuk, Albert Einstein College of Medicine, 1300 Morris

Park Avenue, Bronx, NY 10461, USA. e-mail: josh.nosanchuk@einstein. yu.edu
Radioimmunotherapy is the targeted delivery of cytocidal radiation to cells via specific antibody. Although mature for the treatment of cancer, RIT of infectious diseases is in preclinical development. However, as there is an obvious and urgent need for novel approaches to treat infectious diseases, RIT can provide us with a powerful approach to combat serious diseases, including invasive fungal infections. For example, RIT has proven more effective than standard amphotericin B for the treatment of experimental cryptococcosis. This review will discuss the concepts of RIT, its applications for infectious diseases, and the strides made to date to bring RIT of infectious diseases to fruition. Finally, we will discuss the potential of PAN-FUNGAL RIT, the targeting of conserved fungal cell surface antigens by RIT, as a treatment modality for fungi prior to the formal microbiological identification of the specific pathogen. In sum, RIT provides a mechanism for the targeted killing of drug susceptible or resistant fungi irrespective of the host immune status and may dramatically reduce the length of therapy currently required for many invasive fungal diseases.

Keywords: Histoplasma capsulatum, Cryptococcus neoformans, Candida albicans, antibody, heat shock protein 60, beta-glucan, melanin

\section{INTRODUCTION}

Fungal diseases are increasingly common major causes of human disease, especially in immunocompromised individuals and hospitalized patients with underlying medical conditions (Patterson, 2005; Caston-Osorio et al., 2008; Richardson and Lass-Florl, 2008; Pfaller and Diekema, 2010). In fact, since 1979 there has been a $>200 \%$ increase in the annual number of cases of invasive fungal infections (IFI) in the United States. IFIs rates have risen in large part due enhanced interventions in intensive care units (ICUs), increased survival rates of individuals with immunodeficiencies, and the increased administration of potent therapeutics, chemotherapeutics, and biologicals, that compromise the immune responses of our patients. This increase is best exemplified by the example of Candida spp.: Candida were once infrequent causes of invasive disease, whereas they are currently the fourth leading cause of nosocomial bloodstream infection in the United States, responsible for $8-15 \%$ of all such hospital acquired infections. However, despite the increased prevalence of many mycotic diseases, there remains an enormous gap in knowledge and our current therapeutic armamentarium all too often fails to eradicate these insidious pathogens.

Although they have powerful activities, the number of available medications for mycoses is significantly less than for bacterial diseases. At present, there are three main medication categories for IFI: azoles (fluconazole, itraconazole, voriconazole, and posaconazole), polyenes (primarily formulations of amphotericin B), and echinocandins (caspofungin, micafungin, and anidulafungin). Notably, both the azoles and polyenes target cell membrane sterols, with azoles inhibiting sterol synthesis and the polyenes purportedly disrupting the membrane structure. The echinocandins inhibit cell wall production by interfering with beta-1,3-glucan synthesis. In addition to these drugs, flucytosine, an antimetabolite, is utilized primarily in combination with amphotericin B for the treatment of cryptococcosis. Notably, the echinocandins are the last new class of antifungal drug, with caspofungin gaining FDA approval in by the FDA in 2001. Unfortunately, there is no antifungal medication poised to enter clinical medicine for the foreseeable future. Hence, there is a consensus that new approaches are needed to combat IFI.

Radioimmunotherapy (RIT) uses antigen-antibody interactions to deliver cytocidal amounts of ionizing radiation to specific cell targets. Currently, RIT is clinically utilized in the treatment of primary, refractory, and recurrent non-Hodgkin lymphoma using the radiolabeled mAbs Zevalin ${ }^{\circledR}$ and Bexxar ${ }^{\circledR}$. It is important to note that RIT offers several significant advantages over standard antifungal therapy. Firstly, RIT delivers lethal radiation, such that it does not merely interfere with a single cellular pathway but completely destroys targeted cells. As such, RIT is less subject to drug resistance mechanisms. Moreover, RIT is cidal in immunologically compromised individuals as the nuclides are equally able to destroy cell targets in immunologically intact individuals or those with HIV or other immunodeficiencies, either primary or drug induced. RIT does not suffer the drug-drug 
interactions that clinically trouble clinicians caring for complex patients, such as azole or echinocandin interactions with commonly prescribed immunosuppressive drugs, like cyclosporine or tacrolimus. Finally, in contrast to weeks, months, or years required for the treatment of certain mycoses with standard antifungals, RIT may permit single dose or a limited number of doses to combat fungal diseases.

What are the barriers for translating RIT into treatment approaches for infectious diseases? Cell surface antigens are well defined for diverse pathogens, including viruses, bacteria, parasites, and fungi. Moreover, monoclonal antibodies exist that target microbial cell surface antigens. Additionally, the technology for linking radionuclides to mAbs is well established, so the approaches can be readily translated from oncology into infectious diseases. Additionally, the US hospitals that are now regularly using RIT to treating cancer patients are fully equipped for initiating Infectious Diseases RIT. Included in this ability, imaging of patients receiving RIT to ascertain the targeting of radiolabeled mAbs in Infectious Diseases RIT can be readily achieved using portable imaging equipment that is standard in these hospitals. Hence, the time is "now" for developing RIT to combat IFI.

\section{RIT OF INFECTIOUS DISEASES}

Our laboratories were the first to demonstrate that microorganismspecific mAb-RIT is highly effective for the treatment of experimental fungal, bacterial, and viral infections, as well as virally induced cancers (Table 1). Although the initial RIT work utilized Cryptococcus neoformans for proof-of-principle studies in 2003 (Dadachova et al., 2003), RIT of bacterial and viral pathogens also has rapidly progressed. In 2004, we established the feasibility of RIT for invasive bacterial infection using a mouse pneumococcal disease model (Dadachova et al., 2004a). An IgM isotype mAb to serotype 8 Streptococcus pneumoniae capsular polysaccharide was conjugated to the alpha-particle emitter Bismuth-213 $\left({ }^{213} \mathrm{Bi}\right)$ and we showed that an $80-\mu \mathrm{Ci}$ dose was sufficient to protect $60 \%$ of animals from an otherwise lethal challenge. More recently, in 2009 , mAbs to the protective or lethal antigens of Bacillus anthracis labeled with either ${ }^{213} \mathrm{Bi}$ or the beta-particle emitter rhenium-188 $\left({ }^{188} \mathrm{Re}\right)$ were shown to prolong the survival of mice infected with $B$. anthracis Stern bacterial cells, but not spores (Rivera et al., 2009).
Treatment with ${ }^{213} \mathrm{Bi}$ was more effective than when less powerful beta-particles were used.

In 2006, HIV infected cells were found to be effectively targeted by RIT (Dadachova et al., 2006a). Radiolabeling of antibody to the HIV-1 envelope glycoproteins gp120 and gp41 with ${ }^{213} \mathrm{Bi}$ or ${ }^{188}$ Re selectively killed HIV infected cells in vitro and eliminated the majority of HIV infected cells injected into SCID mice. Notably, the mAb to gp41 is fully human. It is possible that, used in combination with anti-retroviral drugs, that RIT may provide a means to eradicate HIV infection (Casadevall et al., 2007). RIT has also proven to be highly effective in the treatment of virally induced cancers (Dadachova et al., 2007). Using a human papillomavirus (HPV) type 16-associated cervical cancer model in mice, RIT with a human mAb targeting the HPV16 E6 antigen significantly impeded tumor growth compared to cancers in control animals (Wang et al., 2007). Interestingly, RIT targeting cancer cells expressing low levels of HPV16 E6 was also successful (Phaeton et al., 2010a). Furthermore, pre-treatment of the cervical tumors with a proteosome inhibitor, MG-132, and unlabeled antibody to E6 resulted in an increase in the levels of E6 for targeting by ${ }^{188}$ Re-labeled $\mathrm{mAb}$ and enhanced the efficacy of RIT (Phaeton et al., 2010b). These studies also suggest that RIT could potentially eradicate infected cells prior to their malignant transformation. In addition to cervical cancer, RIT proof-of-principle experiments with HPV-associated head and neck squamous cell carcinoma suggest that this modality may be effective for this difficult to combat disease (Harris et al., 2011).

\section{RIT OF CRYPTOCOCCOSIS}

Cryptococcus neoformans is a yeast-like encapsulated fungus with a global distribution that is responsible for $\sim 600,000$ deaths annually (Park et al., 2009), particularly in individuals with HIV infection. As noted above, the first RIT experiments published in 2003 utilized C. neoformans as a model organism (Dadachova et al., 2003). The initial studies with C. neoformans demonstrated that a $\mathrm{mAb}$ specific for glucuronoxylomannan (GXM), the major component of the pathogen's polysaccharide capsule, radiolabeled with either ${ }^{213} \mathrm{Bi}$ or ${ }^{188} \mathrm{Re}$ efficiently killed cryptococcal cells in vitro and significantly reduced fungal burdens in and prolonged the survival of lethally infected mice (Dadachova et al., 2003). Most impressively, $60 \%$ of infected mice treated with $100 \mu \mathrm{Ci}^{213} \mathrm{Bi}$ were alive at

Table 1 | List of monoclonal antibodies that have been used in experimental studies of radioimmunotherapy for infectious diseases.

\begin{tabular}{|c|c|c|c|}
\hline Antibody & Isotype & Antigen recognized & Reference or source \\
\hline D11 & IgM, human & Serotype 8 pneumococcal polysaccharide & Dadachova et al. (2004a), Zhong et al. (1999) \\
\hline $7.5 \mathrm{G}$ & IgG2b, mouse & B. anthracis protective antigen & Rivera et al. $(2006,2009)$ \\
\hline 10F4 & $\operatorname{lgG} 1$, mouse & B. anthracis protective antigen & Rivera et al. $(2006,2009)$ \\
\hline 14FA & $\operatorname{lgG} 2 b$, mouse & B. anthracis lethal antigen & Rivera et al. (2009) \\
\hline $18 \mathrm{~B} 7$ & $\operatorname{lgG} 1$, mouse & GXM, cryptococcal polysaccharide & Dadachova et al. (2003, 2004b, 2006b), Bryan et al. (2009, 2010) \\
\hline 246-D (cluster I) & $\operatorname{lgG} 1$, human & HIV gp41 & Dadachova et al. (2006a) \\
\hline C1-P5 & $\operatorname{lgG} 1$, mouse & HPV E6 & Wang et al. (2007), Phaeton et al. (2010a,b), Harris et al. (2011) \\
\hline $2 \mathrm{G8}$ & lgG2b, mouse & Beta $(1,3)$ glucan & Torosantucci et al. (2009), Rachini et al. (2007) \\
\hline $4 \mathrm{E} 12$ & $\operatorname{lgG} 2 a$, mouse & Hsp60 & Guimaraes et al. (2009) \\
\hline $6 \mathrm{D} 2$ & IgM, mouse & Melanin & Rosas et al. (2000) \\
\hline B11 & $\lg M$ & Fungal glucosyl ceramide & Rhome et al. (2011) \\
\hline
\end{tabular}


day 75 after RIT. Importantly, systemically infected mice tolerated activities of up to $150 \mu \mathrm{Ci}$ of mAb labeled with either nuclide with platelet counts normalizing by 14 days post-treatment (Dadachova et al., 2004b). The reduction in platelets in the infected mice was similar to that seen in the setting of cancer therapy (Sharkey et al., 1997; Behr et al., 1999). Additionally, these mice had no evidence of pulmonary fibrosis 5 months after receiving RIT. The lack of severe toxicities due to radiation in the mice with cryptococcosis treated with RIT is presumably due to the high specificity of the mAb.

Chemotherapy for severe cryptococcosis is based on the administration of amphotericin B. However, RIT was recently shown to be more effective than amphotericin B for the treatment of disseminated cryptococcosis (Bryan et al., 2010). In the murine model examined, amphotericin B was able to prolong survival, but fungal burdens were not significantly reduced. In contrast, RIT significantly reduced the fungal burdens in the tissues examined, regardless of the melanization state of the yeast cell. This is an important observation as $C$. neoformans can produce melanin along the yeast cell wall and this pigment can protect the fungus from injury from external radiation (Nosanchuk and Casadevall, 2006; Dadachova et al., 2008). Interestingly, $\sim 10$ times less mAb $18 \mathrm{~B} 7$ conjugated to ${ }^{213} \mathrm{Bi}$ was required for killing melanized C. neoformans yeast cells than ${ }^{188}$ Re conjugated antibody (Bryan et al., 2010), demonstrating the superior penetrating power of the alpha-particle versus the beta-particle emitter. Combining RIT with amphotericin B was more effective than amphotericin B alone (Bryan et al., 2010). However, combination therapy was less potent than RIT alone if melanized fungal cells were used for the infectious challenge. It is not clear whether this result is due to the potential toxicities of the amphotericin or the inflammatory responses that are induced upon infection with melanized yeast cells (Mednick et al., 2005).

Although drug resistance is a concern in the treatment of fungal infections, there is no literature on pathogen resistance to RIT. Nevertheless, experiments have been performed to assess whether resistance can develop (Bryan et al., 2009). C. neoformans yeast cells were harvested from infected mice that received RIT and subjected to treatment with ${ }^{213} \mathrm{Bi}$ or ${ }^{188} \mathrm{Re}$ conjugated mAbs. Notably, the RIT and radiation naive $C$. neoformans yeast cells were similarly radiosensitive to the radiolabeled mAbs. Additionally, no differences in survival occurred comparing mice infected with RIT $C$. neoformans to radiation naive yeast cells.

\section{MECHANISM}

Although the precise mechanism of action is not known, there are major radiobiological probabilities for the effectiveness of RIT. The first and most targeted effect occurs by a "direct hit," when the radiolabeled antibody binds to a cell and the emitted particles kill the cell (Figure 1A). However, "cross-fire" effects, killing of a cell by particles emitted from a radiolabeled antibody bound to a distant cell, can be equally efficient (Figure 1B). Both of these mechanisms lead to cell apoptosis and cell cycle redistribution (Macklis, 2004). It is also highly probable, that a radiolabeled antibody could kill by both of these processes. Notably, the "cross-fire" mechanism would be expected to play a large role in combating fungal biofilm diseases or situations where some fungi are extracellular, whilst others are intracellular.

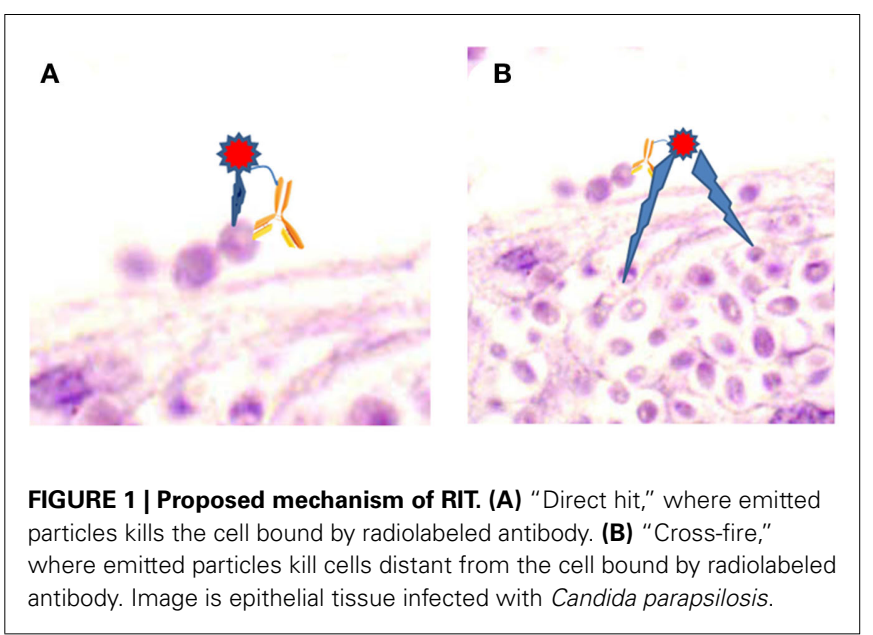

Certain mechanisms of radiation effects on fungi have been studied using C. neoformans. C. neoformans yeast cells can be directly killed by gamma, beta, and alpha radiation in a dose and time dependent manner (Bryan et al., 2008). Notably, although membrane permeability did occur, it does not appear to be the primary cause of death. Gamma radiation induced significantly more membrane disruption than either beta or alpha radiation. All forms of radiation induced cellular apoptosis, although external gamma radiation and ${ }^{188} \mathrm{Re}$-labeled antibody induced more apoptosis than ${ }^{213} \mathrm{Bi}$-labeled antibody. However, ${ }^{213} \mathrm{Bi}$-labeled antibody significantly reduced the metabolic activity of the yeast cells, whereas the other forms of radiation did not. Hence, the cellular effects of radiation on yeast are dependent on dose and time as well as the form of radiation administered. In a more recent study, gamma radiation, as well as UV and visible light, were shown to affect ATP levels in C. neoformans yeast cells, and this effect was more pronounced in melanized compared to non-melanized cells (Bryan et al., 2011a). Another study assessed whether "direct hit" or "cross-fire" predominated in the killing of C. neoformans yeast cells (Dadachova et al., 2006b). As expected, given the power of alpha radiation, ${ }^{213} \mathrm{Bi}$-labeled antibody resulted in significant "direct hit" killing. However, ${ }^{213} \mathrm{Bi}$-labeled antibody bound to heat-killed yeast cells also led to "cross-fire" fungicidal effects. In contrast, the killing with the lower energy beta-particles produced by the ${ }^{188}$ Re-labeled antibody was primarily due to "cross-fire."

\section{RIT TOXICITIES}

As briefly described above, the primary toxicity identified in our RIT experiments is a modest thrombocytopenia that resolves within 14 days post therapy, which is similar to that seen in RIT in cancer patients (Macklis and Pohlman, 2006). Importantly, thrombocytopenia prediction models are developed for RIT and dose-fractionated RIT strategies can be implemented to minimize the nadir and duration of the thrombocytopenia (Shen et al., 2002). Importantly, since microbial cells in otherwise sterile sites are foreign to hosts, the microbes display antigens that are distinct from those expressed by host cells. These distinct microbial antigens are the primary targets for infectious diseases RIT. In contrast, RIT of cancers targets tumor-associated antigens that are also expressed on normal cells, which clearly increases toxicity 
risks. Therefore, in addition to leading to a theoretically higher therapeutic index in infectious diseases RIT relative to that for cancer, RIT of infectious diseases should have a lower toxicity risk than RIT for cancer.

There is an important issue of "free antigen" as pathogens release/secrete many substances, including proteins, carbohydrates, and lipids. Hence, antibody directed to released or potentially secreted antigen has the potential to produce off-target effects. Previously, we have studied this issue in murine cryptococcosis, since $C$. neoformans releases large amounts of capsular polysaccharide and we were concerned that the radiolabeled antibody to the capsule would interact with non-organism associated polysaccharide. However, we determined that radiolabeled antibody had higher affinity for mice-associated polysaccharide than for its soluble form (Dadachova et al., 2003). Nevertheless, unlabeled antibody can be administered prior to RIT in order to potentially clear circulating antigens.

\section{BIOFILMS}

Biofilms are complex communities of pathogens that adhere to one another and are typically surrounded by a matrix of extracellular materials, particularly exopolysaccharides. Fungal biofilms can be especially tenacious and can exacerbate disease by leading to increased drug resistance or enhanced adherence to foreign bodies or tissues. RIT using mAb to C. neoformans GXM conjugated to ${ }^{213} \mathrm{Bi}$ can effectively damage cryptococcal biofilms ( $50 \%$ reduction in metabolic activity; Martinez et al., 2006). Notably, beta and gamma forms of radiation did not have a significant effect on the fungal biofilms. It was not determined whether RIT of biofilms synergized with antifungal drugs, as the alterations in biofilm could enhance drug penetration facilitating the activities of the drugs on fungal cells. Hence, RIT may be a means for combating fungal biofilms with or without concomitant antifungal drugs.

\section{CONCEPT OF PAN-FUNGAL RIT}

To dramatically drive the treatment of infectious diseases forward with RIT, we need to make a "quantum leap" by identifying antigens shared by major IFI-causing pathogens (PAN-FUNGAL antigens) in order to deliver RIT without the need for specific mycological diagnosis or fear of drug resistance. To this end, we have been exploring the possibility of targeting common cell wall associated antigens, which also happen to constitute major virulence factors for these fungi. We have begun exploring the utility of four cell surface antigens. The majority of fungal cells, yeast, and hyphal, display beta-glucans on their cell surface and Cassone and colleagues have generated a mAb to beta-glucan that provides significant protection against Candida albicans, C. neoformans, and Aspergillus fumigatus in animal models if given prior to infection (Torosantucci et al., 2005, 2009; Rachini et al., 2007). Heat shock protein 60 (Hsp60) is a key regulator of virulence in Histoplasma capsulatum and mAbs directed to this protein are protective in murine histoplasmosis (Guimaraes et al., 2009). Recently, we demonstrated that mAbs to H. capsulatum Hsp60 also bound other pathogenic fungal species (Bryan et al., 2011b), but does not react with human Hsp60 (Guimaraes et al., 2009). Melanin is present in the cell wall of diverse human fungal pathogens and a monoclonal to fungal melanin has been shown to bind $C$. neoformans, H. capsulatum, Aspergillus spp., C. albicans, Scytalidium dimidiatum, Sporothrix schenckii, Paracoccidioides brasiliensis, Coccidioides posadasii, and Blastomyces dermatitidis (reviewed in Nosanchuk and Casadevall, 2006; Taborda et al., 2008). Glucosylceramide is another common exposed cell surface antigen on fungi and a mAb to glucosylceramide has been shown to bind glucosylceramide in C. neoformans and C. albicans, but not mammalian glucosylceramide (Rhome et al., 2011).

Selected mAbs to these four surface antigens were used for radiolabeling and tested for their capacity to kill C. neoformans and C. albicans (Bryan et al., 2011b). ${ }^{213}$ Bi-labeled $>90 \%$ of mAbs 2G8, IgG2b to beta-galactan, and 4E12, IgG2a to Hsp60, but the labeling efficiency of mAb 6D2, IgM to melanin, was only $30 \%$. ${ }^{213} \mathrm{Bi}$ did not effectively label $\mathrm{mAb} \mathrm{B} 11$, IgM to glucosylceramide, but the $\mathrm{mAb}$ was labeled by ${ }^{188} \mathrm{Re}$. Radiolabeled $\mathrm{mAb}$ to beta-glucan killed $100 \%$ of $C$. neoformans yeast cells, whereas $C$. albicans pseudohyphal cells were significantly more resistant as the treatment produced a 30\% reduction in CFUs. Targeting Hsp60 produced $>90 \%$ reductions in CFUs of both C. neoformans and C. albicans. The poor ${ }^{213} \mathrm{Bi}$ radiolabeling of the $\mathrm{mAb}$ to melanin resulted in the delivery of three to four times less radiation than the ${ }^{213} \mathrm{Bi}$-labeled $\mathrm{mAb}$ to beta-glucan and $\mathrm{Hsp} 60$, and $\sim 4 \mu \mathrm{Ci} / 5$ million yeast cells was the maximal radiation delivered resulting in only 15 and $22 \%$ reduction for C. albicans and C. neoformans, respectively, but the decrease was only significant for $C$. neoformans. The ${ }^{188}$ Re-labeled mAb to glucosylceramide decreased the CFU of $C$. neoformans by $40 \%$. Hence, we have demonstrated that we can radiolabel diverse antibodies to distinct conserved surface antigens and deliver cytocidal radiation to important fungal pathogens by RIT. It is noteworthy that the efficacy of killing was achieved with significantly lower $(\sim 1,000$ times $)$ concentrations than were required for biological efficacy with unlabeled antibody, such as with mAb 2G8 (Torosantucci et al., 2009). Moreover, the data demonstrated that the powerful alpha particles from ${ }^{213} \mathrm{Bi}$ efficiently killed fungal cells even when there were relatively few binding sites on their cell surface ( $~ 5 \%$ mAb binding). Additional experimentation is required to develop more efficient labeling of IgM isotype mAbs and perhaps improve the labeling of IgG isotype mAbs. Nevertheless, the PAN-FUNGAL concept is validated by these proof-of-principle experiments and further study is clearly warranted.

\section{DISCUSSION}

The drive to develop RIT of infectious diseases is fueled by the facts that (1) our current antifungal armamentarium often fails to eradicate IFI, (2) the last new category of antifungals was FDA approved in 2001, (3) no new antifungal drugs are close to clinical availability, and (4) RIT is a means to specifically target microbes in a cytocidal manner, irrespective of the host immune status, current drug regimen, or potential resistance mechanisms of the microbe. With RIT of fungi, especially with PAN-FUNGAL RIT, we can potentially reduce treatment durations, prevent the toxicities of the prolonged administration of (possibly ineffective) antifungals, minimize concerns of drug-drug interactions, permit the preservation of current immunomodulating medications (i.e., for transplant patients), and eliminate the need for a specific mycological 
diagnosis. Given the well developed applications of RIT in Oncology, the barriers to initiating RIT for infectious diseases, especially IFIs that continue to have unacceptably high mortality rates, are indeed surmountable. The work to date demonstrates that we have in hand a diverse set of specific mAbs and mAbs with reactivity to several leading fungal pathogens that can be harnessed for RIT. We are hopeful that the upcoming years will see a broader acceptance of this approach and a rapid growth in pre-clinical work that will

\section{REFERENCES}

Behr, T. M., Béhé, M., Stabin, M. G., Wehrmann, E., Apostolidis, C., Molinet, R., Strutz, F., Fayyazi, A., Wieland, E., Gratz, S., Koch, L., Goldenberg, D. M., and Becker, W. (1999). High-linear energy transfer (LET) alpha versus low-LET beta emitters in radioimmunotherapy of solid tumors: therapeutic efficacy and dose-limiting toxicity of 213Biversus 90Y-labeled CO17-1A Fab' fragments in a human colonic cancer model. Cancer Res. 59, 2635-2643.

Bryan, R., Jiang, Z., Friedman, M., and Dadachova, E. (2011a). The effects of gamma radiation, UV and visible light on ATP levels in yeast cells depend on cellular melanization. Fungal Biol. 115, 945-949.

Bryan, R. A., Guimaraes, A. J., Hopcraft, S., Jiang, Z., Bonilla, K., Morgenstern, A., Bruchertseifer, F., Del Poeta, M., Torosantucci, A., Cassone, A., Nosanchuk, J. D., Casadevall, A., and Dadachova, E. (2011b). Toward developing a universal treatment for fungal disease using radioimmunotherapy targeting common fungal antigens. Mycopathologia. doi: 10.1007/s11046011-9476-9. [Epub ahead of print].

Bryan, R. A., Huang, X., Morgenstern, A., Bruchertseifer, F., Casadevall, A., and Dadachova, E. (2008). Radiofungicidal effects of external gamma radiation and antibodytargeted beta and alpha radiation on Cryptococcus neoformans. Antimicrob. Agents Chemother. 52, 2232-2235.

Bryan, R. A., Jiang, Z., Howell, R. C., Morgenstern, A., Bruchertseifer, F., Casadevall, A., and Dadachova, E. (2010). Radioimmunotherapy is more effective than antifungal treatment in experimental cryptococcal infection. J. Infect. Dis. 202, 633-637.

Bryan, R. A., Jiang, Z., Huang, X., Morgenstern, A., Bruchertseifer, F., Sellers, R., Casadevall, A., and Dadachova, E. (2009). Radioimmunotherapy is effective against high-inoculum Cryptococcus neoformans infection in mice and does not select for radiation-resistant cryptococcal cells. Antimicrob. Agents Chemother. 53, 1679-1682.

Casadevall, A., Goldstein, H., and Dadachova, E. (2007). Targeting host cells harbouring viruses with radiolabeled antibodies. Expert Opin. Biol. Ther. 7, 595-597.

Caston-Osorio, J. J., Rivero, A., and Torre-Cisneros, J. (2008). Epidemiology of invasive fungal infection. Int. J. Antimicrob. Agents 32(Suppl. 2), S103-S109.

Dadachova, E., Bryan, R. A., Howell, R. C., Schweitzer, A. D., Aisen, P., Nosanchuk, J. D., and Casadevall, A. (2008). The radioprotective properof its chemical composition, stable radical presence and spatial arrangement. Pigment Cell Melanoma Res. 21, 192-199.

Dadachova, E., Burns, T., Bryan, R. A., Apostolidis, C., Brechbiel, M. W., Nosanchuk, J. D., Casadevall, A., and Pirofski, L. (2004a). Feasibility of radioimmunotherapy of experimental pneumococcal infection. Antimicrob. Agents Chemother. 48, 1624-1629.

Dadachova, E., Bryan, R. A., Frenkel, A., Zhang, T., Apostolidis, C., Nosanchuk, J. S., Nosanchuk, J. D., and Casadevall, A. (2004b). Evaluation of acute hematologic and long-term pulmonary toxicities of radioimmunotherapy of Cryptococcus neoformans infection in murine models. Antimicrob. Agents Chemother. 48, 1004-1006.

Dadachova, E., Nakouzi, A., Bryan, R. A., and Casadevall, A. (2003). Ionizing radiation delivered by specific antibody is therapeutic against a fungal infection. Proc. Natl. Acad. Sci. U.S.A. 20, 20

Dadachova, E., Patel, M. C., Toussi, S., Apostolidis, C., Morgenstern, A., Brechbiel, M. W., Gorny, M. K., Zolla-Pazner, S., Casadevall, A., and Goldstein, H. (2006a). Targeted killing of virally infected cells by radiolabeled antibodies to viral proteins. PLoS Med. 3, e427. doi:10.1371/journal.pmed.0030427

Dadachova, E., Bryan, R. A., Apostolidis, C., Morgenstern, A., Zhang, T., Moadel, T., Torres, M., Huang, ties of fungal melanin are a function

allow the translation of RIT from the basic science laboratory to our patients.

\section{ACKNOWLEDGMENTS}

E. Dadachova is a Sylvia and Robert S. Olnick Faculty Scholar in Cancer Research and is supported by the NIH grant R56 AI06050706. Joshua D. Nosanchuk is in part supported by NIH grant AI52733.

X., Revskaya, E., and Casadevall, A. (2006b). Interaction of radiolabeled antibodies with fungal cells and components of the immune system in vitro and during radioimmunotherapy for experimental fungal infection. J. Infect. Dis. 193, 1427-1436.

Dadachova, E., Wang, X. G., and Casadevall, A. (2007). Targeting the virus with radioimmunotherapy in virus-associated cancers. Cancer Biother. Radiopharm. 22, 303-308.

Guimaraes, A. J., Frases, S., Gomez, F. J., Zancope-Oliveira, R. M. and Nosanchuk, J. D. (2009). Monoclonal antibodies to heat shock protein 60 alter the pathogenesis of Histoplasma capsulatum. Infect. Immun. 77, 1357-1367.

Harris, M., Wang, X. G., Jiang, Z. Goldberg, G. L., Casadevall, A., and Dadachova, E. (2011). Radioimmunotherapy of experimental head and neck squamous cell carcinoma (HNSCC) with E6-specific antibody using a novel HPV-16 positive HNSCC cell line. Head Neck Oncol. 3,9 .

Macklis, R. M. (2004). How and why does radioimmunotherapy work? Int. J. Radiat. Oncol. Biol. Phys. 59, 1269-1271.

Macklis, R. M., and Pohlman, B. (2006). Radioimmunotherapy for non-Hodgkin's lymphoma: a review for radiation oncologists. Int. J. Radiat. Oncol. Biol. Phys. 66, 833-841.

Martinez, L. R., Bryan, R. A., Apostolidis, C., Morgenstern, A., Casadevall, A., and Dadachova, E. (2006) Antibody-guided alpha radiation effectively damages fungal biofilms. Antimicrob. Agents Chemother. 50, 2132-2136.

Mednick, A. J., Nosanchuk, J. D., and Casadevall, A. (2005). Melanization of Cryptococcus neoformans affects lung inflammatory responses during cryptococcal infection. Infect. Immun. 73, 2012-2019.

Nosanchuk, J. D., and Casadevall, A. (2006). Impact of melanin on microbial virulence and clinical resistance to antimicrobial compounds.
Antimicrob. Agents Chemother. 50 3519-3528.

Park, B. J., Wannemuehler, K. A., Marston, B. J., Govender, N., Pappas, P. G., and Chiller, T. M. (2009). Estimation of the current global burden of cryptococcal meningitis among persons living with HIV/AIDS. AIDS 23, 525-530.

Patterson, T. F. (2005). Advances and challenges in management of invasive mycoses. Lancet 366, 1013-1025.

Pfaller, M. A., and Diekema, D. J. (2010). Epidemiology of invasive mycoses in North America. Crit. Rev. Microbiol. 36, 1-53.

Phaeton, R., Harris, M., Jiang, Z., Wang, X. G., Einstein, M. H., Goldberg, G. L., Casadevall, A., and Dadachova, E. (2010a). Radioimmunotherapy with an antibody to the HPV16 E6 oncoprotein is effective in an experimental cervical tumor expressing low levels of E6. Cancer Biol. Ther. 10, 1041-1047.

Phaeton, R., Wang, X. G., Einstein, M. H., Goldberg, G. L. Casadevall, A., and Dadachova, E. (2010b). The influence of proteasome inhibitor MG132, external radiation, and unlabeled antibody on the tumor uptake and biodistribution of (188)re-labeled antiE6 C1P5 antibody in cervical cancer in mice. Cancer 116(4 Suppl.), 1067-1074.

Rachini, A., Pietrella, D., Lupo, P., Torosantucci, A., Chiani, P., Bromuro, C., Proietti, C., Bistoni, F., Cassone, A., and Vecchiarelli, A. (2007). An anti-beta-glucan monoclonal antibody inhibits growth and capsule formation of Cryptococcus neoformans in vitro and exerts therapeutic, anticryptococcal activity in vivo. Infect. Immun. 75, 5085-5094.

Rhome, R., Singh, A., Kechichian, T., Drago, M., Morace, G., Luberto, C., and Del Poeta, M. (2011). Surface localization of glucosylceramide during Cryptococcus neoformans infection allows targeting as a potential antifungal. PLoS ONE 6, e15572. doi:10.1371/journal.pone. 0015572 
Richardson, M., and Lass-Florl, C. (2008). Changing epidemiology of systemic fungal infections. Clin. Microbiol. Infect. 14(Suppl. 4), 5-24.

Rivera, J., Nakouzi, A., Abboud, N., Revskaya, E., Goldman, D., Collier, R. J., Dadachova, E., and Casadevall, A. (2006). A monoclonal antibody to Bacillus anthracis protective antigen defines a neutralizing epitope in domain 1. Infect. Immun. 74, 4149-4156.

Rivera, J., Nakouzi, A. S., Morgenstern, A., Bruchertseifer, F., Dadachova, E., and Casadevall, A. (2009). Radiolabeled antibodies to Bacillus anthracis toxins are bactericidal and partially therapeutic in experimental murine anthrax. Antimicrob. Agents Chemother. 53, 4860-4868.

Rosas, A. L., Nosanchuk, J. D., Feldmesser, M., Cox, G. M., McDade, H. C., and Casadevall, A. (2000). Synthesis of polymerized melanin by Cryptococcus neoformans in infected rodents. Infect. Immun. 68, 2845-2853.

Sharkey, R. M., Blumenthal, R. D., Behr, T. M., Wong, G. Y., Haywood,
L., Forman, D., Griffiths, G. L., and Goldenberg, D. M. (1997). Selection of radioimmunoconjugates for the therapy of wellestablished or micrometastatic colon carcinoma. Int. J. Cancer 72, 477-485.

Shen, S., Duan, J., Meredith, R. F., Buchsbaum, D. J., Brezovich, I. A., Pareek, P. N., and Bonner, J. A. (2002). Model prediction of treatment planning for dose-fractionated radioimmunotherapy. Cancer 94(4 Suppl.), 1264-1269.

Taborda, C. P., da Silva, M. B., Nosanchuk, J. D., and Travassos, L. R. (2008). Melanin as a virulence factor of Paracoccidioides brasiliensis and other dimorphic pathogenic fungi: a minireview. Mycopathologia 165, 331-339.

Torosantucci, A., Bromuro, C., Chiani, P., De Bernardis, F., Berti, F., Galli, C., Norelli, F., Bellucci, C., Polonelli, L., Costantino, P., Rappuoli, R., and Cassone, A. (2005). A novel glyco-conjugate vaccine against fungal pathogens. J. Exp. Med. 202, 597-606.
Torosantucci, A., Chiani, P., Bromuro, C., De Bernardis, F., Palma, A. S. Liu, Y., Mignogna, G., Maras, B. Colone, M., Stringaro, A., Zamboni, S., Feizi, T., and Cassone, A. (2009). Protection by anti-betaglucan antibodies is associated with restricted beta-1,3 glucan binding specificity and inhibition of fungal growth and adherence. PLOS ONE 4, e5392. doi:10.1371/journal.pone. 0005392

Wang, X. G., Revskaya, E., Bryan, R. A., Strickler, H. D., Burk, R. D., Casadevall, A., and Dadachova, E. (2007). Treating cancer as an infectious disease - viral antigens as novel targets for treatment and potential prevention of tumors of viral etiology. PLoS ONE 2, e1114. doi:10.1371/journal.pone.0001114

Zhong, Z., Burns, T., Chang, Q., Carroll, M., and Pirofski, L. (1999). Molecular and functional characteristics of a protective human monoclonal antibody to serotype 8 Streptococcus pneumoniae capsular polysaccharide. Infect. Immun. 67 4119-4127.
Conflict of Interest Statement: The authors declare that the research was conducted in the absence of any commercial or financial relationships that could be construed as a potential conflict of interest.

Received: 10 October 2011; accepted: 28 December 2011; published online: 12 January 2012.

Citation: Nosanchuk JD and Dadachova E (2012) Radioimmunotherapy of fungal diseases: the therapeutic potential of cytocidal radiation delivered by antibody targeting fungal cell surface antigens. Front. Microbio. 2:283. doi 10.3389/fmicb.2011.00283

This article was submitted to Frontiers in Fungi and Their Interactions, a specialty of Frontiers in Microbiology.

Copyright $($ C) 2012 Nosanchuk and Dadachova. This is an open-access article distributed under the terms of the Creative Commons Attribution Non Commercial License, which permits noncommercial use, distribution, and reproduction in other forums, provided the original authors and source are credited. 